\title{
Suppression of SHP-1 promotes corticospinal tract sprouting and functional recovery after brain injury
}

\author{
T Tanaka ${ }^{1,2}$, Y Fujita $^{1,2}$, M Ueno $^{1,2}$, LD Shultz $^{3}$ and T Yamashita ${ }^{*, 1,2}$
}

Reorganization of spared neural network connections is one of the most important processes for restoring impaired function after brain injury. However, plasticity is quite limited in the adult brain due to the presence of inhibitory molecules and a lack of intrinsic neuronal signals for axonal growth. Src homology 2-containing phosphatase (SHP)-1 has been shown to have a role in axon growth inhibition. Here, we tested the hypothesis that SHP-1 negatively affects axonal reorganization. We observed that unilateral motor cortex injury led to increased expression and activity of SHP-1 in the contralesional cortex. In this model, corticospinal axons originating from the contralesional cortex sprouted into the denervated side of the cervical spinal cord after injury. We observed that the number of sprouting fibers was increased in SHP-1-deficient heterozygous viable motheaten $\left(+/ m e^{v}\right)$ mice, which show reduced SHP-1 activity, and in wild-type mice treated with an SHP inhibitor. Motor function recovery of impaired forelimb was enhanced in $+/ m e^{v}$ mice. Collectively, our results indicate that downregulation of SHP-1 activity promotes corticospinal tract sprouting and functional recovery after brain injury.

Cell Death and Disease (2013) 4, e567; doi:10.1038/cddis.2013.102; published online 4 April 2013

Subject Category: Neuroscience

Brain injuries often cause severe motor deficits. Compensatory axonal reorganization in spared neural networks is one of the most important steps to restore impaired functions. ${ }^{1-3}$ However, function is not completely recovered due to the lack of ability to regenerate or reorganize adult central nervous system (CNS) axons. Numerous studies indicate that many inhibitory or repulsive guidance cues might restrict axonal plasticity in the CNS. Myelin-derived proteins, such as myelinassociated glycoprotein (MAG), Nogo and oligodendrocyte myelin glycoprotein (OMgp) potently inhibit axonal plasticity. ${ }^{4,5}$ Although these proteins are structurally distinct, they all bind to the Nogo receptor and paired immunoglobulin-like receptor $B$ (PIR-B), which transduce signals that inhibit axon growth. ${ }^{6,7}$ PIR-B recruits Src homology 2-containing phosphatase-1 (SHP-1), an intracellular cytoplasmic protein tyrosine phosphatase (PTP) ${ }^{8-10}$ SHP-1, a member of the SHP family of PTPs, is recruited to PIR-B upon MAG stimulation and is required for MAG-induced neurite growth inhibition and dephosphorylation of tropomyosin receptor kinase $B\left(\right.$ TrkB). ${ }^{11}$ TrkB encodes a receptor for brain-derived neurotrophic factor (BDNF), ${ }^{12,13}$ and BDNF/TrkB signaling is important for axonal regeneration and rewiring. ${ }^{14,15} \mathrm{We}$ previously demonstrated that inhibition of SHP-1 signaling enhanced axonal regeneration after optic nerve injury. ${ }^{11}$ These studies suggest that SHP-1 is a key signaling molecule that mediates axonal growth inhibition via myelin-dependent signals and inactivation of growth factor signaling. However, it remains unclear whether SHP-1 inhibition can facilitate axonal plasticity and functional recovery after brain injury.

In this study, we asked whether downregulation of SHP-1 activity enhances corticospinal tract (CST) sprouting and functional recovery after unilateral cortical injury in SHP-1deficient heterozygous viable motheaten $\left(+/ m e^{v}\right)$ mice or in wild-type $(+/+)$ mice treated with a SHP inhibitor. The current study examined the role of SHP-1 on CST sprouting from the contralesional motor cortex, which is thought to contribute to motor function recovery. ${ }^{3,15-19}$

\section{Results}

CST formation in cervical cord of wild-type and $+/ \mathbf{m e}^{v}$ mice. We first examined CST fibers in cervical cord of intact wild-type and $+/ m e^{v}$ (8-weeks-old) mice. We labeled the CST by injecting the anterograde tracer, biotinylated dextran amine (BDA), into the right motor cortex and analyzed the CST axons that recrossed the midline into the right side of the cervical cord. BDA-positive midline-crossing fibers of the CST were slightly observed in intact mice. This result was consistent with previous observation showing that the CST midline-crossing fibers were observed in sham-operated

\footnotetext{
${ }^{1}$ Department of Molecular Neuroscience, Graduate School of Medicine, Osaka University, Osaka, Japan; ${ }^{2}$ Core Research for Evolutional Science and Technology (CREST), Japan Science and Technology Agency (JST), Tokyo, Japan and ${ }^{3}$ The Jackson Laboratory, Bar Harbor, ME, USA

*Corresponding author: T Yamashita, Department of Molecular Neuroscience, Graduate School of Medicine, Osaka University, 2-2 Yamadaoka, Suita-shi, Osaka 565-0871, Japan. Tel: 816 68793661; Fax: 816 68793669; E-mail: yamashita@ molneu.med.osaka-u.ac.jp

Keywords: SHP-1; corticospinal tract; axonal sprouting; functional recovery; brain injury

Abbreviations: SHP, Src homology 2-containing phosphatase; $m e^{v}$, viable motheaten; CNS, central nervous system; MAG, myelin-associated glycoprotein; OMgp, oligodendrocyte myelin glycoprotein; PIR-B, paired immunoglobulin-like receptor B; PTP, protein tyrosine phosphatase; TrkB, tropomyosin receptor kinase B; BDNF, brain-derived neurotrophic factor; CST, corticospinal tract; BDA, biotinylated dextran amine; NSC-87877, 8-hydroxy-7-(6-sulfonaphthalen-2-yl)diazenyl-quinoline-5sulfonic acid; PKC $\gamma$, protein kinase C $\gamma$; SHP-1L, long form of SHP-1; Ptpn6, PTP non-receptor type 6; PBS, phosphate-buffered saline; GAPDH, glyceraldehyde 3phosphate dehydrogenase; $\mathrm{Ct}$, cycle threshold

Received 15.10.12; revised 7.2.13; accepted 27.2.13; Edited by D Bano
} 
mice. ${ }^{19}$ The number of CST midline-crossing axons was not significantly different between the wild-type and $+/ m e^{v}$ mice (Supplementary Figures 1a and b).

SHP-1 expression in cortical neurons. We next investigated SHP-1 expression in the cerebral cortex. We focused on corticospinal motor neurons, which are located in layer $\mathrm{V}$ of the motor cortex and regulate motor function. Cortical sections were immunostained with anti-NeuN (a neuronal marker), anti-Ctip2 (a marker for layer $\mathrm{V}$ neurons) and antiSHP-1 antibodies. In wild-type mice, SHP-1 was expressed in cortical neurons, including the layer $\mathrm{V}$ neurons (Figures 1a and b). The amount of SHP-1 protein in the cerebral cortex was further examined in wild-type and $+/ m e^{v}$ mice using western blot analysis, and quantitative analysis confirmed that SHP-1 expression was significantly decreased in the cortex of $+/ m e^{v}$ mice (Figures $1 c$ and $d$ ). These data indicate that SHP-1 expression is decreased in the cerebral cortex in $+/ m e^{v}$ mice compared with wild-type littermates.

Expression and phosphatase activity of SHP-1 are increased in the contralesional cortex after injury. We employed a murine model of cortical injury to elucidate the role of SHP-1 signaling in neuronal reorganization and functional recovery. This model induces collateral sprouting of CST axons from the contralesional cortex to innervate the cervical cord, to compensate for the lost function. ${ }^{15,19} \mathrm{We}$ hypothesized that SHP-1 regulates this axonal plasticity and tested it by assessing the expression and phosphatase activity of SHP-1 in the contralesional cortex following injury.

Real-time PCR analysis was performed on CDNA obtained from the contralesional sensorimotor cortex. SHP-1 mRNA expression was increased after the injury was compared with intact cerebral cortex, and the highest values were observed on post-injury days 3-7 (Figure 2a). The increase began to decline on day 14. However, we failed in detecting statistically significant upregulation of SHP-1 protein level after cortical injury, although there seems to be some tendency of upregulation (Figures $2 b$ and $c$ ). We then compared SHP-1 protein expression in the contralesional cortex between wildtype and $+/ m e^{v}$ mice and found that it was lower in $+/ m e^{v}$ mice at baseline compared with wild-type mice (Figures $2 d$ and e), and injury did not induce a significant increase in SHP1 protein expression in $+/ m e^{v}$ mice (Figure $2 \mathrm{e}$ ). Collectively, these results demonstrate that SHP-1 expression is increased in the contralesional cortex in wild-type but not in $+/ m e^{v}$ mice after cortical injury.

We further examined SHP-1 phoshotyrosine phosphatase activity in the contralesional sensorimotor cortex. The relative phosphatase activity of SHP-1 was increased after injury compared with intact cerebral cortex (Figure 2f). Therefore, SHP-1 activity is increased in the contralesional motor cortex following brain injury.

Suppressing SHP-1 activity promotes CST axonal sprouting. The above results support the hypothesis that SHP-1 might have a role in restricting axonal plasticity after brain injury. We next examined the effect of blocking SHP-1 activity on axonal reorganization and functional recovery after cortical injury in wild-type versus $+/ m e^{v}$ mice. In addition, the effects of treatment of wild-type mice with the SHP inhibitor 8-hydroxy-7-(6-sulfonaphthalen-2-yl)diazenylquinoline-5-sulfonic acid (NSC-87877) were determined.

We first evaluated lesion volume and CST destruction to ensure the injury protocol was effective. Cortical ablation resulted in complete destruction of the sensorimotor cortex 4 weeks after the injury, and Nissl staining (Supplementary Figure 2a) confirmed that there were no significant differences in brain lesion volume between wild-type and $+/ m e^{v}$ mice (Supplementary Figure 2b) or saline- and NSC-87877-treated mice (Supplementary Figure 2c). To determine whether the effects of ablation mainly affected the CST, the cervical spinal cord was stained for protein kinase $\mathrm{C} \gamma(\mathrm{PKC} \gamma)$, a marker for CST, 4 weeks after the injury. In sham control mice, PKC $\gamma$ immunoreactivity was present bilaterally in the dorsal CST of the cervical cord (data not shown). In lesioned mice, however, $\mathrm{PKC} \gamma$ immunoreactivity was extremely low in the right dorsal CST originating from the injured left motor cortex (Supplementary Figure 2d). There were no significant differences in degenerated CST between the lesioned wild-type and $+/ m e^{v}$ mice (Supplementary Figure $2 \mathrm{e}$ ) or between saline- and NSC-87877-treated mice (Supplementary Figure 2f). These data indicate that the cortical ablation model is appropriate for studying CST destruction.

We then investigated whether downregulation of SHP-1 activity promoted CST sprouting. We first performed cortical injury in $+/ m e^{v}$ mice, in which SHP-1 PTP catalytic activity was genetically decreased and observed that unilateral motor cortex lesions induce neuroanatomical changes in the contralesional CST (Figure 3a). Two weeks after surgery, we labeled the CST with BDA, an anterograde tracer, and analyzed the CST axons that crossed the midline into the denervated side of the cervical cord at 4 weeks after the injury. The number of midline-crossing axons of the uninjured CST across to the denervated side was significantly increased in $+/ m e^{v}$ mice compared with wild-type mice (Figures $3 b-1, c-1$ and d). In addition, the number of axon fibers and axon branches in the denervated side was significantly increased in $+/ m e^{v}$ mice (Figures 3b-2, b-3, c-2, c-3, e and f).

We next examined the site-specific effects of SHP-1 downregulation, using the inhibitor NSC-87877, which was continuously infused into the forelimb region of the contralesional motor cortex for 14 days after injury. Consistent with the observations in $+/ m e^{v}$ mice, the number of midline-crossing axons and axon fibers in the denervated side was significantly increased in NSC-87877-treated wild-type mice compared with saline-treated mice (Figures $3 g$ and $h$ ). However, the number of axon branches of uninjured CST in the denervated side was not significantly different in NSC-87877-treated mice compared with control (Figure $3 \mathrm{i})$. These results indicate that decreasing SHP-1 activity can promote CST axonal reorganization in vivo.

Behavioral recovery is enhanced in $+/ m e^{v}$ mice after cortical injury. We assessed the functional recovery by employing two motor tests beginning on the second postoperative day and then once a week for 4 weeks. First, the cylinder test was used to assess the independent use of an affected forelimb that contacted the interior cylinder wall during a rear movement. ${ }^{20}$ On day 2 , both groups showed 
a

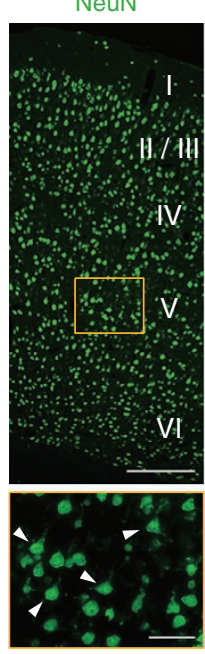

SHP-1

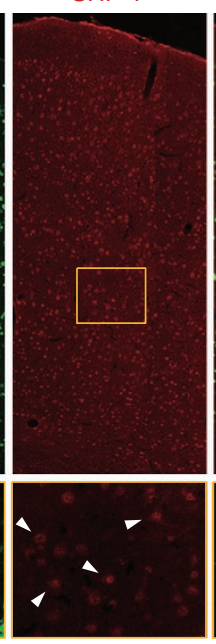

Merge

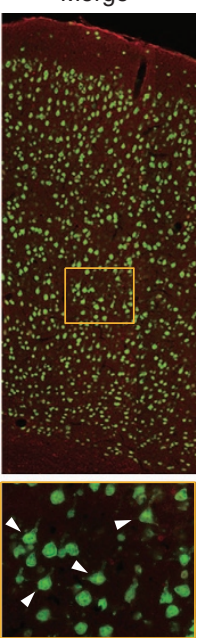

b

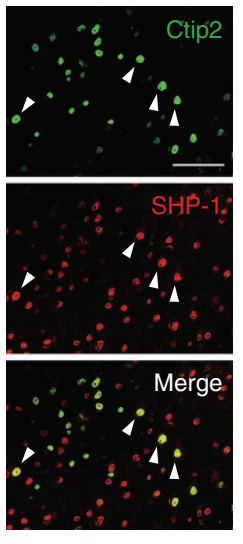

C

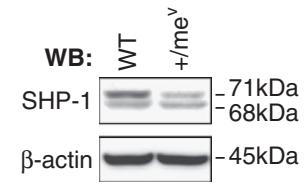

d

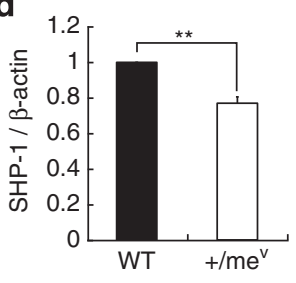

Figure 1 SHP-1 is expressed in cortical neurons. (a) NeuN (green) and SHP-1 (red) staining in the adult cerebral cortex (upper panels) and layer V of cerebral cortex (lower panels). Arrowheads indicate the expression of SHP-1 in the layer V neurons. Scale bars: upper, $200 \mu \mathrm{m}$ : lower, $50 \mu \mathrm{m}$. (b) Ctip2 (green) and SHP-1 (red) staining in the adult motor cortex. Arrowheads indicate the expression of SHP-1 in the Ctip2-positive neurons. Scale bar, $50 \mu \mathrm{m}$. (c) SHP-1 expression in wild-type and $+/ \mathrm{me}^{v}$ mice. The expression level of SHP-1 was examined by western blot analysis. (d) SHP-1 signal intensity was quantified by densitometry and normalized to $\beta$-actin. Data are presented as mean \pm S.E.M $\left(n=5\right.$, each group). ${ }^{* *} P<0.01$, Student's $t$-test

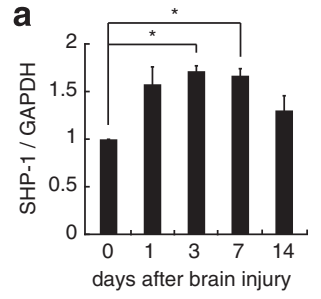

d

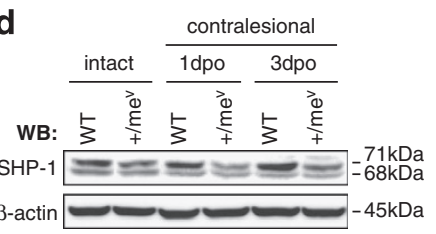

b
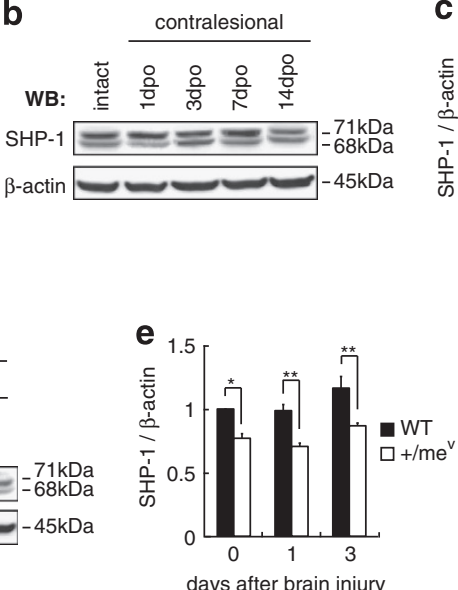

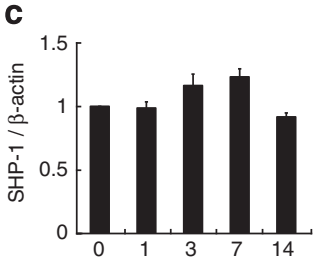

days after brain injury

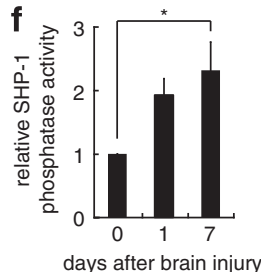

Figure 2 The expression and phosphatase activity of SHP-1 are increased in the contralesional cortex after injury. (a-c) Relative expression of SHP-1 in the contralesional cortex. SHP-1 expression was examined by real-time PCR (a) and western blot (b and $\mathbf{c})$. Contralesional cortices were isolated at the indicated days post-operation (dpo). Data are presented as mean \pm S.E.M. (PCR, $n=3-4$; western blot, $n=5$, each group). ${ }^{*} P<0.05$, one-way ANOVA followed by Tukey-Kramer test. (d and e) SHP-1 protein expression in the contralesional cortex of wild-type and $+/ m e^{v}$ mice after injury. (f) Relative phosphatase activity of SHP-1 in the contralesional cortex. Data are presented as mean \pm S.E.M. ( $n=5$, each group). ${ }^{*} P<0.05,{ }^{* *} P<0.01$, one-way ANOVA followed by Tukey-Kramer test

marked deficits in impaired right forelimb use. The frequency of impaired forelimb use gradually increased over 4 weeks, indicating some degree of spontaneous recovery from the neurological deficit. The $+/ m e^{v}$ mice tended to use their impaired forepaws more frequently compared with wild-type controls (Figure 4a). Next, the grid-walk test was used to assess skilled walking during a free exploration on a wire grid with square holes. ${ }^{20,21}$ We analyzed the percentage of missed steps of the impaired right forelimb out of 50 steps. On day 2 postinjury, mice in all the groups showed marked deficits in their ability to grasp the wire and to accurately place the impaired forelimb. Thereafter, $+/ m e^{v}$ mice showed enhanced recovery compared with wild-type controls (Figure 4b). These results indicate that suppressing SHP-1 activity promotes functional recovery after brain injury.

\section{Discussion}

Here, we have shown that suppression of SHP-1 activity by a genetic approach or following treatment with an SHP inhibitor significantly increased CST sprouting and enhanced spontaneous motor function recovery after cortical injury. Thus, inhibition of SHP-1 activity is important for axonal plasticity in the adult CNS and the following recovery. 


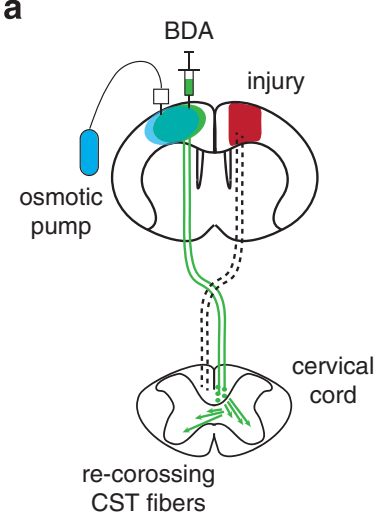

NSC-87877

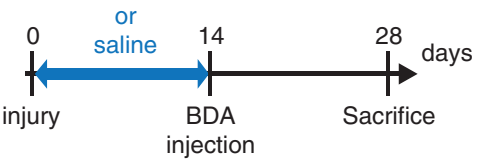

b
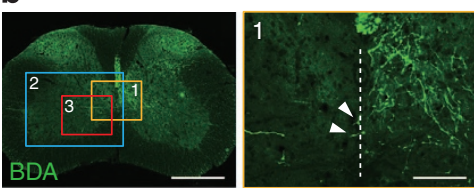

2
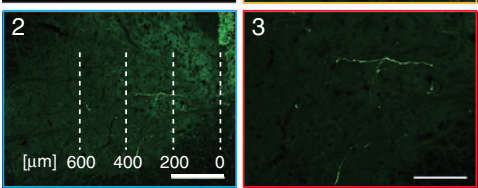

C
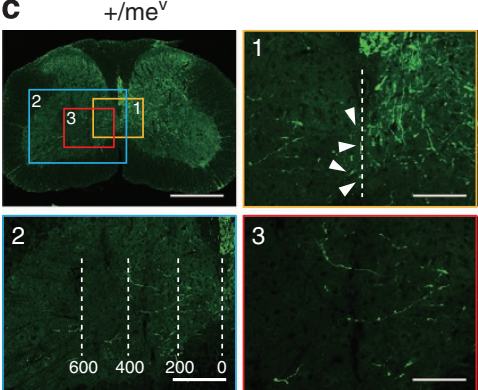
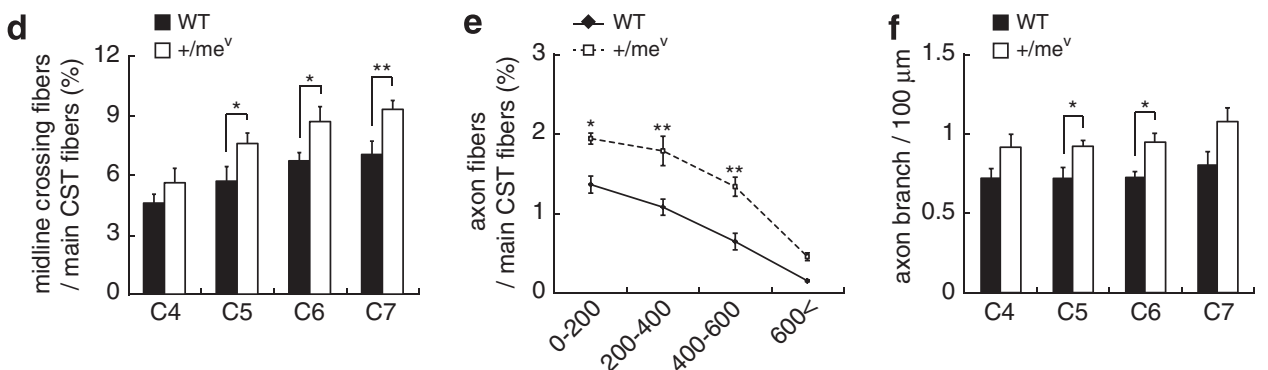

distance of midline $[\mu \mathrm{m}]$
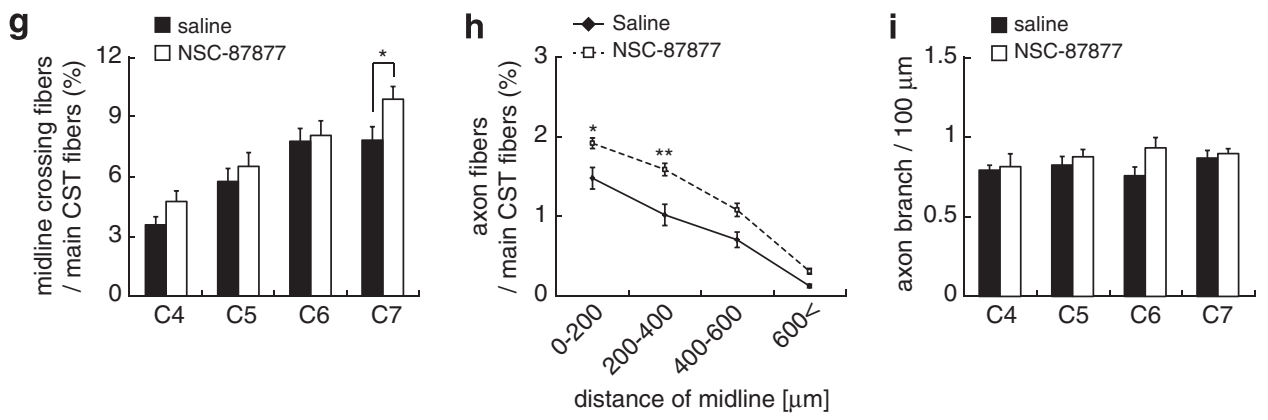

Figure 3 Inhibition of SHP-1 activity promotes CST sprouting after cortical injury. (a) Schematic illustration of the cortical injury model in this study. Cortical injury to the sensorimotor cortex (red) damages the CST (dotted lines). Saline or NSC-87877 (SHP inhibitor) was continuously infused for 14 days. The anterograde tracer BDA was injected into the contralesional motor cortex to label the intact CST 2 weeks after injury. The green arrows indicate sprouting axons of the intact CST crossing the midline into the denervated side. (b and $\mathbf{c}$ ) Transverse cervical cord sections showing BDA-labeled CST axons (green) 28 days after cortical injury in wild-type (b) and $+/ m e^{v}$ mice (c). Scale bars, $500 \mu \mathrm{m}$. (1) Arrowheads indicate midline-crossing CST fibers. Scale bars, $100 \mu \mathrm{m}$. (2) The white dashed lines indicate the distance from midline, and axonal fibers of uninjured CST in the denervated side were quantified in each area. Scale bars, $200 \mu \mathrm{m}$. (3) Axon branches of uninjured CST fibers in the denervated side. Scale bars, $100 \mu \mathrm{m}$. (d and g) Quantitative data of the number of CST axons crossing the midline in wild-type and $+/ \mathrm{me}^{v}$ mice (d) or saline- and NSC-87877-treated mice (g). Data are presented as mean \pm S.E.M. (wild-type, $n=8$; $+/ m e^{v}, n=10$; saline, $n=7$; NSC-87877, $n=7$ ). ${ }^{*} P<0.05$, Student's $t$-test. (e and $\mathbf{h}$ ) Quantitative data of axon fibers of the uninjured CST in the denervated side at C7 in wild-type and $+/ m e^{v}$ mice (e) or saline- and NSC-87877-treated mice (h). Data are presented as mean \pm S.E.M. (wild-type, $n=5 ;+/ \mathrm{me}^{v}, n=5$; saline, $n=7$; NSC-87877, $n=7$ ). ${ }^{*} P<0.05,{ }^{* \star} P<0.01$, one-way ANOVA followed by Tukey-Kramer test. (f and i) Quantitative data of the number of axon branches in the denervated side in wild-type and $+/ m e^{v}$ mice (f) or saline- and NSC-87877-treated mice (i). The branches were quantified 4 weeks after injury. Data are presented as mean \pm S.E.M. (wild-type, $n=5 ;+/ m e^{v}, n=5$; saline, $n=5$; NSC-87877, $n=5$ ). ${ }^{*} P<0.05,{ }^{* \star} P<0.01$, Student's $t$-test 

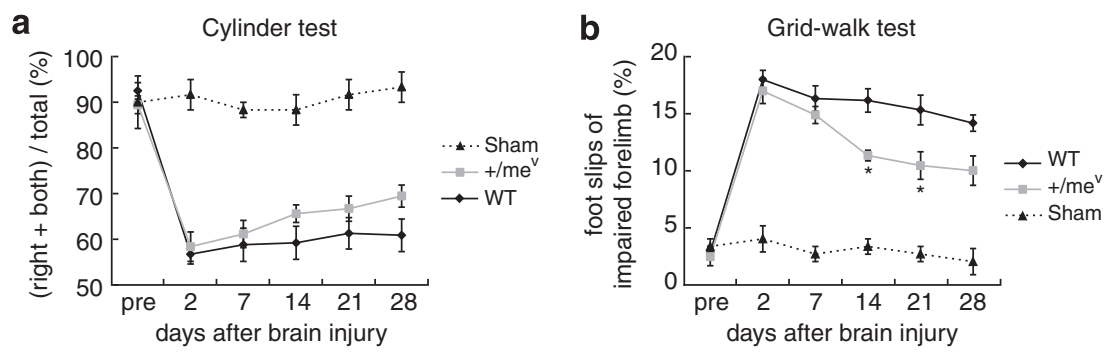

Figure 4 Downregulation of SHP-1 activity enhances functional motor recovery after brain injury. (a) Cylinder test scores in wild-type and $+/ m e^{v}$ mice after cortical injury. (b) Grid-walk test scores in wild-type and $+/ m e^{v}$ mice after cortical injury. Data are presented as mean \pm S.E.M. (wild-type, $n=12 ;+/ m e^{v}, n=9$; sham, $n=3$ ). ${ }^{*} P<0.05$, two-way repeated measures ANOVA followed by Tukey-Kramer test

SHP inactivation is known to activate downstream pathway of Trk, and inhibit other signaling molecules, such as PIR-B. SHP is recruited to PIR-B, a receptor for myelin inhibitors MAG, Nogo and OMgp, through MAG stimulation, which is critical for inhibitory signaling of axonal outgrowth. ${ }^{7,11} \mathrm{We}$ recently demonstrated that PIR-B deletion did not promote axonal regeneration and CST sprouting after spinal cord injury or traumatic brain injury. ${ }^{19,22}$ Notably, knockdown of PIR-B's downstream signaling molecule SHP-1 promotes axonal regeneration via TrkB activation after optic nerve injury. ${ }^{11}$ TrkB is a known neurotrophin receptor, and its activation often leads to axonal elongation. ${ }^{12,13}$ We further demonstrated that BDNF/TrkB signaling was crucial for CST rewiring after traumatic brain injury. ${ }^{15}$ These observations suggest that SHP-1 suppression not only reduces inhibitory signals, it also enhances signals that induce axonal regeneration. Thus, it can be assumed that these dichotomous actions of SHP-1 would enhance CST sprouting and functional motor recovery in $+/ m e^{v}$ and NSC-87877-treated mice in the present study.

We observed SHP-1 expression in cortical neurons, including layer $\mathrm{V}$, of adult mice. A previous study also reported that SHP-1 expression was localized to pyramidal neurons of the hippocampus and cortex, as well as astrocytes and oligodendrocytes, in rodent CNS. ${ }^{23,24}$ Therefore, it is conceivable that intrinsic SHP-1 activity in the corticospinal neurons directly affects axonal growth. In this view, SHP-1 may be an important intrinsic factor that determines neuronal capacity for axonal growth in the adult CNS. The effect of cortical-specific SHP-1 inhibition would further test this notion. It should also be noted that there was no significant difference in the number of midline-crossing fibers between WT and $+/ m e^{v}$ mice. Therefore, SHP-1 may have no or little role in midline-crossing of the CST during the developmental stage.

In the genetic approach, we used heterozygous $m e^{v}$ mice because homozygous $m e^{v}$ mice have a greatly shortened lifespan. We confirmed decreased SHP-1 expression in $+/ m e^{v}$ cortex, indicating that the model is appropriate for analyzing the role of SHP-1. The results are consistent with previous data by Kozlowski et al. ${ }^{25}$ showing that SHP-1 expression was reduced in $+/ m e^{v}$ mice compared with wildtype mice. Importantly, they further demonstrated that SHP-1 phosphatase activity was decreased in various $m e^{v}$ mice tissues. ${ }^{25}$ Their findings suggest that in addition to decreased expression, SHP-1 proteins are enzymatically impaired by the $m e^{v}$ mutation.
SHP-1 western blotting revealed two proteins with molecular masses of 68 and $71 \mathrm{kDa}$ (Figure 1c). A $71 \mathrm{kDa}$ long form of SHP-1 (SHP-1L), is longer than the $68 \mathrm{kDa}$ SHP-1 and differs by 66 amino acids at the $\mathrm{C}$-terminus due to alternative transcript splicing and a subsequent reading frame shift. ${ }^{26}$ Some cytokines, such as IL- 6 and IFN- $\beta$, have been shown to induce SHP-1L expression. ${ }^{27}$ However, additional information on it is still limited. Thus, the precise regulatory mechanisms of differential SHP-1 expression after injury remain unclear and will be determined in future studies.

Enhanced spontaneous recovery after injury was observed in $+/ m e^{v}$ mice, particularly during the late phase in the behavioral tests, and this observation corresponded with CST sprouting which increases 2 weeks after injury. ${ }^{15}$ Therefore, SHP-1 suppression in the contralesional cortex would be an effective way to enhance neuronal plasticity and subsequent functional recovery. To assess CST-dependent motor functions, we performed grid-walk test, cylinder test. The motor function measured by the grid-walk test may be, at least partly, dependent on CST, as the similar task, ladder walk test, is dependent on the function of the CST. ${ }^{15}$ This is substantiated by the observation that selective transection of CST leads to reimpairment of recovered function in ladder walk test after hemicortical ablation. Other group also reported that CST is required for grid-walk task as well as cylinder test. ${ }^{21}$ In this study, the surviving CST on the lesioned side might also be involved in restored motor function. Indeed, previous studies have shown that significant bilateral innervation of the intact CST is well correlated with functional recovery in models of cortical photothrombosis and focal cortical infarct, ${ }^{17,28}$ and these rewired CST axons actually contribute to functional recovery after cortical injury. ${ }^{15}$ Therefore, enhanced CST sprouting induced by suppression of SHP-1 activity would enable functional recovery. In addition, it is suggested that there are at least two important processes for sprouting CST fibers to make a neural connection in the denervated side; crossing the midline (elongation) and then branching in the denervated side. ${ }^{15}$ Both processes are required for neural network formation and the underlying mechanisms are different. Our data in the present study suggest that SHP-1 is involved in both of these plastic changes. However, it is noted that reorganization of multiple tracts, such as corticorubral, corticoreticular and corticocortical pathways, might also have roles in functional recovery by inhibiting SHP-1 activity, although we focused on CST in 
the present study. Our findings may help establish an effective therapeutic method that facilitates recovery following CNS injuries.

\begin{abstract}
Materials and Methods
Mice. All experiments were conducted in accordance with the Osaka University Medical School Guide for the Care and Use of Laboratory Animals and were approved by the institutional committee of Osaka University. C57BL/6J mice (8-weeks-old) were purchased from Charles River Japan (Tokyo, Japan). The mice were bred and maintained in the Institute of Experimental Animal Sciences, Osaka University Graduate School of Medicine. C57BL/6J mice carrying the viable motheaten mutation at the PTP, non-receptor type 6 (Ptpn6) locus (Ptpn6 $6^{\text {me- }}$ ), abbreviated as $m e^{v}$, which have severely impaired expression and catalytic activity of the SHP-1 protein tyrosine phosphatase, also termed PTP $1 C,{ }^{25}$ were originally obtained from the Jackson Laboratory (Bar Harbor, ME, USA). Mice used in these studies were propagated by mating $+/ m e^{v}$ heterozygotes, thereby producing $m e^{v} / m e^{v},+/ m e^{v}$ and $+I+$ offspring. The $+I+$ mice were used as wild-type controls. The $m e^{v} / m e^{v}$ mice develop systemic autoimmunity and have a shortened lifespan. Therefore $+/ m e^{v}$ mice that have reduced SHP-1 catalytic activity but do not develop autoimmune disease were used in these experiments. Genotypes at the Ptpn6 locus were determined by PCR. PCR amplifications were performed by 35 cycles of denaturation at $94^{\circ} \mathrm{C}$, annealing at $54^{\circ} \mathrm{C}$ and extension at $72{ }^{\circ} \mathrm{C}$, followed by a final extension at $72{ }^{\circ} \mathrm{C}$. The following primer were used: 5'-GGTATTGAACAAGGACCAAGG-3' (common), 5'-GAGGTGGAGAAAGGCCG GGT-3' (wild-type) and $5^{\prime}$-GAGGTGGAGAAAGGCCGGGA-3' (me'). PCR products were electrophoresed in Tris acetate ethylenediaminetetraacetic acid electrophoresis buffer containing 3\% agarose (Nippon Gene, Toyama, Japan) and visualized by staining with ethidium bromide.
\end{abstract}

Surgical procedures for unilateral cortical ablation. Unilateral lesions of the primary motor cortex were induced in 4-weeks-old male mice (wildtype, $n=63 ;+/ m e^{v}, n=29$ ) as described previously Omoto et al. ${ }^{19}$ Briefly, the skull was exposed with a midline skin incision, and bone overlying the left motor cortex was removed. Cortical ablation (depth: $1.0 \mathrm{~mm}$ in the motor cortex) was performed by aspiration with a pipette. ${ }^{19}$ The injured area covered primary forelimb and hindlimb motor area of cerebral cortex. ${ }^{29}$ The wound was sutured, and the animals were housed in standard cages with free access to food and water. Age-matched, intact mice (wild-type, $n=20 ;+/ m e^{v}, n=10$ ) and shamoperated mice (wild-type, $n=3$ ) underwent the same surgical procedures except for cortical ablation.

Drug treatment and intra-cortical injection. We purchased NSC-87877, a potent inhibitor of SHP-1 and SHP-2, from Calbiochem (San Diego, CA, USA). NSC-87877 was dissolved in dimethyl sulfoxide as a stock solution $(20 \mathrm{mM})$ and brought to its final concentration $(200 \mu \mathrm{M})$ with $0.9 \%$ saline immediately before the experiment. Mice were anesthetized and secured on a stereotaxic apparatus. A stainless steel cannula (Brain infusion kit 3; Alzet, Cupertino, CA, USA) connected to a microosmotic pump (model 1004; Alzet) was implanted in the forelimb area of the right contralesional motor cortex $1.0 \mathrm{~mm}$ anterior and $1.0 \mathrm{~mm}$ lateral to the bregma, $1.0 \mathrm{~mm}$ depth from the skull surface) immediately after the injury. NSC-87877 (200 $\mu \mathrm{M}$ in saline) was infused continuously for 14 days (days $0-14 ; 0.11 \mu / / h$ ). Saline was administered in control animals.

Anterograde CST labeling. Two weeks after surgery, mice were anesthetized as described above and placed on a stereotaxic frame. The skull overlying the right primary motor cortex was carefully removed with a drill. For mice outfitted with a stainless steel cannula connected to a micro-osmotic pump, the skull was removed after cannula removal. To visualize the uninjured CST, the anterograde tracer BDA (molecular weight, $10000 ; 10 \%$ in phosphate-buffered saline (PBS); Invitrogen, Carlsbad, CA, USA) was injected at three sites within the forelimb motor area as guided by a functional motor cortex map $(1.0 \mathrm{~mm}$ lateral to bregma; $1.0 \mathrm{~mm}$ lateral to bregma, $0.5 \mathrm{~mm}$ anterior; $1.5 \mathrm{~mm}$ lateral to bregma and $0.5 \mathrm{~mm}$ anterior; $0.5 \mathrm{~mm}$ depth; $0.6 \mu \mathrm{l} / \mathrm{site}) .{ }^{29}$ For the uninjured CST tracing experiment, mice were killed 2 weeks after injection with BDA (4 weeks after cortical ablation). The mice were transcardially perfused with $4 \%$ paraformaldehyde in PBS. The cervical spinal cord was dissected, postfixed in the same fixatives, and immersed overnight in PBS containing $30 \%$ sucrose. The sections were blocked for $4 \mathrm{~h}$ in PBS supplemented with $0.3 \%$ Triton X-100 (PBS-T) and then incubated for $2 \mathrm{~h}$ with Alexa Fluor 488-conjugated streptavidin $(1: 400$; Invitrogen, Grand Island, NY, USA) in PBS-T.

Quantitative analysis of uninjured CST sprouting. CST projections from the contralesional primary motor cortex to denervated cervical cord gray matter were quantitatively analyzed with fluorescence microscopy (BX51; Olympus, Tokyo, Japan) at $\times 200$ magnification. We counted the number of BDA-positive midline-crossing fibers in serial transverse sections of the cervical spinal cord (C4-C7) at $\times 200$ in the midline of 20 sections per cervical segment, and the value was normalized to the number of main CST fibers in the most rostral portion of C4. ${ }^{15,19,30}$ The number of BDA-positive fibers in the dorsal CST was determined from images captured by a laser scanning confocal microscope (FV1000; Olympus) with ImageJ software (National Institutes of Health, Bethesda, MD, USA). Axonal fibers of uninjured CST in the denervated side was evaluated at four defined distances from the midline $(0-200 \mu \mathrm{m}, 200-400 \mu \mathrm{m}, 400-600 \mu \mathrm{m}$, $600<\mu \mathrm{m}$, Figures $3 \mathrm{e}$ and $\mathrm{h}$ ). The number of axon fibers and axon branches in the denervated side were counted at a magnification of $\times 100$ in 10 sections per cervical segment. The number of axonal fibers was normalized to the number of main CST fibers at the C4 level (\% crossing fibers). The number of axonal branches in the denervated side was analyzed in 3-4 long axons in each section (20 $\mu \mathrm{m}$ thick) every $100 \mu \mathrm{m}^{15}$

Immunohistochemistry. The mice were transcardially perfused with $4 \%$ paraformaldehyde in PBS. The brain and cervical spinal cord were dissected postfixed in the same fixatives, and immersed overnight in PBS containing $30 \%$ sucrose. The brain and cervical cord specimens (C4-C7) were then embedded in Tissue-Tek OCT (Sakura Finetek Europe, Alphen aan den Rijn, the Netherlands) and kept frozen at $-80^{\circ} \mathrm{C}$ until use. Coronal sections ( $20 \mu \mathrm{m}$ thick) of the brain and transverse sections ( $20 \mu \mathrm{m}$ thick) of spinal cord were prepared using a cryostat. Cryosections were rinsed in PBS, blocked with $5 \%$ bovine serum albumin (BSA, Sigma-Aldrich, Saint Louis, MO, USA) and 0.1-0.3\% Triton X-100 in PBS for $1 \mathrm{~h}$ and then incubated with rabbit anti-SHP-1 (1:500; Santa Cruz Biotechnology, Santa Cruz, CA, USA), mouse anti-NeuN (a marker for neurons; 1 : 200; Millipore, Billerica, MA, USA), rat anti-Ctip2 (a marker for layer V neurons; 1:200; Abcam, Cambridge, MA, USA) or rabbit anti-PKC $\gamma$ (1:500; Santa Cruz Biotechnology) antibodies overnight at $4{ }^{\circ} \mathrm{C}$. Alexa 488 anti-mouse IgG (1:1000; Invitrogen), Alexa 488 anti-rat IgG (1:1000; Invitrogen) and Alexa 568 anti-rabbit IgG (1:1000; Invitrogen) were used as secondary antibodies. Images were acquired using a laser scanning confocal microscope (FV-1000; Olympus) or a fluorescent microscope (BX51; Olympus).

Quantification of lesion volume. To assess brain lesion volume, consecutive coronal brain sections ( $20 \mu \mathrm{m}$ thick) were stained with cresyl violet (Nissl stain; Sigma-Aldrich). The area of lesioned structures was expressed as a percentage of the volume of corresponding structures in the contralesional hemispheres in each of 12 sections (spaced $200 \mu \mathrm{m}$ apart). Lesion volume was measured using Photoshop software (Adobe Systems, San Jose, CA, USA), and the percentage of the lesion volume was calculated as (contralesional cortexipsilesional cortex)/contralesional cortex. ${ }^{31}$ To assess CST destruction volume, transverse cervical cord sections $(20 \mu \mathrm{m}$ thick) were stained with an antibody against PKC $\gamma$ (Santa Cruz Biotechnology), a CST marker. ${ }^{32,33}$ The area of injured CST was expressed as a percentage of the PKC $\gamma$ immunoreactive area of the uninjured CST in each of eight sections (C4-C7, spaced $0.5 \mathrm{~mm}$ apart). PKC $\gamma$ staining intensity was measured using ImageJ software, and the percentage of the lesion volume was calculated as: (uninjured CST-the injured CST)/uninjured CST.

Real-Time PCR. Total RNA was extracted from the contralesional cortex with TRIzol reagent (Invitrogen) 1, 3, 7 and 14 days after brain injury and reverse transcribed using a High-Capacity cDNA Reverse Transcription Kit (Applied Biosystems, Foster City, CA, USA). mRNA expression was determined using a 7300 fast real-time PCR system (Applied Biosystems). TaqMan assays were used to quantify SHP-1 (Mm00469153_m1) expression using the TaqMan Gene Expression Master Mix (Applied Biosystems). The relative mRNA expression was calculated after normalizing to glyceraldehyde 3-phosphate dehydrogenase (GAPDH) mRNA expression (Mm99999915_gl). The results of cycle threshold (Ct) values were calculated by the $\Delta \Delta \mathrm{Ct}$ method to obtain the fold differences. 
Western blotting. At 1, 3, 7 and 14 days after the injury, mice were anesthetized and their contralesional cortexes were sampled. Cerebral cortica cells were lysed using a mixture containing $50 \mathrm{mM}$ Tris- $\mathrm{HCl}(\mathrm{pH} 7.4), 150 \mathrm{mM}$ $\mathrm{NaCl}, 1 \% \mathrm{NP}-40,0.1 \% \mathrm{SDS}, 1 \mathrm{mM} \mathrm{Na}_{3} \mathrm{VO}_{4}, 1 \mathrm{mM} \mathrm{NaF}$, and a protease inhibito cocktail (Roche Diagnostics, Indianapolis, IN, USA). The homogenate was centrifuged at $10000 \times g$ for $10 \mathrm{~min}$, and the supernatant was stored at $-80^{\circ} \mathrm{C}$ Protein concentration was measured using a bicinchoninic acid protein assay kit (Pierce/Thermo Scientific, Rockford, IL, USA). Cell lysates were boiled in sample buffer for $5 \mathrm{~min}$, and proteins were separated by SDS-polyacrylamide gel electrophoresis and transferred onto polyvinylidene difluoride membranes (Millipore). The membranes were blocked with 5\% non-fat dry milk in PBS-T and incubated for $1 \mathrm{~h}$ at room temperature. They were then incubated for $1 \mathrm{~h}$ at room temperature or overnight at $4^{\circ} \mathrm{C}$ with anti-SHP-1 (1:500; Santa Cruz Biotechnology) or anti- $\beta$-actin (13E5) antibody (1:2000; Cell Signaling Technology, Danvers, MA, USA) in PBS-T containing $1 \%$ non-fat dry milk. After washing in PBS-T, the membrane was incubated with an HRP-conjugated antirabbit IgG antibody (Cell Signaling Technology). Signals were detected with an enhanced chemiluminescence system (GE Healthcare, Little Chalfont, UK) and quantified using an LAS-3000 image analyzer (Fuji Film, Tokyo, Japan), according to the manufacturer's specifications.

Phosphatase activity assay of SHP-1. At 1 and 7 days after the injury, mice were anesthetized and their contralesional cortices were sampled. Cerebral cortex tissues were lysed using a mixture containing $50 \mathrm{mM}$ HEPES, $0.1 \mathrm{mM}$ EGTA, $0.1 \mathrm{mM}$ EDTA, $120 \mathrm{mM} \mathrm{NaCl}, 0.5 \% \mathrm{NP}-40, \mathrm{pH} 7.5$, a protease inhibitor (Roche Diagnostics) and $1 \mathrm{mM}$ PMSF. The homogenate was centrifuged at $12000 \times g$ for $5 \mathrm{~min}$, and the supernatant was stored at $-80^{\circ} \mathrm{C}$. Phosphatase activity of SHP-1 was measured using an active SHP-1 DuoSet IC ELISA Development (R\&D Systems, Minneapolis, MN, USA). For immunoprecipitation, the mix agarose beads $20 \mu \mathrm{l}$ for each sample was rinsed with $200 \mu \mathrm{l}$ of IC Diluent no. 10 (50 mM HEPES, $0.1 \mathrm{mM}$ EGTA, $0.1 \mathrm{mM}$ EDTA, $120 \mathrm{mM} \mathrm{NaCl}, 0.5 \% \mathrm{NP}-40$, $\mathrm{pH} 7.5$ ), centrifuged briefly, and the supernatant was aspirated off. The packed pellet was putted into the cells lysate, and shaken vigorously at $4{ }^{\circ} \mathrm{C}$ for $3 \mathrm{~h}$. After washing in $200 \mu \mathrm{l}$ of IC Diluent no. 10 and once with $200 \mu \mathrm{l}$ of IC Diluent no. 11 (10 mM HEPES, 0.1 mM EGTA, 0.1 mM EDTA, 0.5\% BSA, $1 \mathrm{mM}$ DTT, pH 7.5), the supernatant was aspirated off. Forty $\mu \mathrm{l}$ of IC Diluent no. 11 and $10 \mu \mathrm{l}$ of diluted 10-fold Tyrosine Phosphatase Substrate I (R\&D Systems) were added to each reaction. The reactions were shaken vigorously at $37^{\circ} \mathrm{C}$ for $30 \mathrm{~min}$. The samples were briefly centrifuged and $50 \mu$ of the supernatant was transferred to wells of an uncoated microplate with $50 \mu \mathrm{l}$ of $50 \mu \mathrm{M}$ Phosphate Standard (R\&D Systems). After $10 \mu \mathrm{l}$ of malachite green reagent A was added into each sample and incubated for $10 \mathrm{~min}$ at room temperature, $10 \mu \mathrm{l}$ of malachite green reagent $\mathrm{B}$ was then added to each well and the plate was incubated for $20 \mathrm{~min}$ at room temperature. Each sample was measured at $\mathrm{A} 620 \mathrm{~nm}$ on a microplate reader (Molecular Devices, Sunnyvale, CA, USA)

\section{Behavioral tests}

Cylinder test. The cylinder test evaluates forelimb use during spontaneous vertical exploration within a cylinder. ${ }^{20}$ Mice were placed in a cylinder $(9 \mathrm{~cm}$ in diameter and $15 \mathrm{~cm}$ high) and recorded with a video camera. We counted the number of contacts with the cylinder wall made by the left and right forelimbs independently and by both forelimbs simultaneously. During a rear movement, if one forelimb (e.g., the right forelimb) made several contacts or dropped immediately after simultaneous contact, both 'both' and 'left' were recorded. A total of 20 movements were recorded during each session. Tes performance was scored as: (impaired right forelimb use + both forelimbs use)/ total use.

Grid-walk test. The grid-walk test assesses the ability to accurately place the forepaws on the rungs of a grid during spontaneous exploration. ${ }^{20,21}$ Mice were placed on a wire grid $(200 \times 240 \mathrm{~mm})$ with $12-\mathrm{mm}$ square holes and allowed to freely explore for $3 \mathrm{~min}$ while performance was recorded with a video camera. The number of foot slips of the impaired right forepaw was assessed as a percentage of the first 50 steps. A foot slip was scored when the paw completely missed a rung (in which case the limb fell between rungs and the animal lost balance) or when the paw was correctly placed on a rung but slipped off while bearing body weight. Neither the cylinder test nor the grid-walk test requires training, but each animal was tested once before surgery to obtain baseline scores.
Statistical analysis. All data are presented as mean \pm S.E.M. SHP-1 protein expression of intact brain between wild-type and $+/ m e^{v}$ mice, lesion volumes, and the number of midline-crossing fibers and axon branches were compared using Student's $t$-tests. The expression and phosphatase activity of SHP-1 after the injury and the percentage of axonal sprouts were compared using one-way ANOVA followed by post hoc Tukey-Kramer tests. Behavioral data were compared using repeated-measure two-ways ANOVA followed by post hoc Tukey-Kramer tests. $P$-values $<0.05$ were considered statistically significant.

\section{Conflict of Interest}

The authors declare no conflict of interest.

Acknowledgements. The authors thank $\mathrm{H}$ Nakagawa, $\mathrm{Y}$ Nakamura, T Itokazu, M Nishibe, $\mathrm{H}$ Ishii and R Muramatsu for their technical assistance and helpful suggestions. This work was supported by Core Research for Evolutional Science and Technology (CREST) from Japan Science and Technology Agency (JST).

1. Nudo RJ. Mechanisms for recovery of motor function following cortical damage. Curr Opin Neurobiol 2006; 16: 638-644.

2. Murphy $\mathrm{TH}$, Corbett D. Plasticity during stroke recovery: from synapse to behaviour. Nat Rev Neurosci 2009; 10: 861-872.

3. Benowitz LI, Carmichael ST. Promoting axonal rewiring to improve outcome after stroke. Neurobiol Dis 2010; 37: 259-266.

4. Yamashita T, Fujitani M, Yamagishi S, Hata K, Mimura F. Multiple signals regulate axon regeneration through the Nogo receptor complex. Mol Neurobiol 2005; 32: 105-111.

5. Giger RJ, Venkatesh K, Chivatakarn O, Raiker SJ, Robak L, Hofer T et al. Mechanisms of CNS myelin inhibition: evidence for distinct and neuronal cell type specific receptor systems. Restor Neurol Neurosci 2008; 26: 97-115.

6. Yiu G, He Z. Glial inhibition of CNS axon regeneration. Nat Rev Neurosci 2006; 7: 617-627.

7. Atwal JK, Pinkston-Gosse J, Syken J, Stawicki S, Wu Y, Shatz $\mathrm{C}$ et al. PirB is a functional receptor for myelin inhibitors of axonal regeneration. Science 2008; 322: 967-970.

8. Wu C, Sun M, Liu L, Zhou GW. The function of the protein tyrosine phosphatase SHP-1 in cancer. Gene 2003; 306: 1-12.

9. Takai T. Paired immunoglobulin-like receptors and their MHC class I recognition. Immunology 2005: 115: 433-440.

10. Syken J, Grandpre T, Kanold PO, Shatz CJ. PirB restricts oculardominance plasticity in visual cortex. Science 2006; 313: 1795-1800.

11. Fujita $Y$, Endo $S$, Takai T, Yamashita T. Myelin suppresses axon regeneration by PIR-B SHP-mediated inhibition of Trk activity. EMBO J 2011; 30: 1389-1401.

12. Kaplan DR, Miller FD. Neurotrophin signal transduction in the nervous system. Curr Opin Neurobiol 2000; 10: 381-391.

13. Reichardt LF. Neurotrophin-regulated signalling pathways. Phil Trans $R$ Soc Lond B Biol Sci 2006; 361: 1545-1564.

14. Hollis ER 2nd, Jamshidi P, Löw K, Blesch A, Tuszynski MH. Induction of corticospinal regeneration by lentiviral trkB-induced Erk activation. Proc Natl Acad Sci USA 2009; 106 : 7215-7220.

15. Ueno M, Hayano $\mathrm{Y}$, Nakagawa $\mathrm{H}$, Yamashita $\mathrm{T}$. Intraspinal rewiring of the corticospinal tract requires target-derived brain-derived neurotrophic factor and compensates lost function after brain injury. Brain 2012; 135: 1253-1267.

16. Chen P, Goldberg DE, Kolb B, Lanser M, Benowitz LI. Inosine induces axonal rewiring and improves behavioral outcome after stroke. Proc Natl Acad Sci USA 2002; 99: 9031-9036.

17. Lee JK, Kim JE, Sivula M, Strittmatter SM. Nogo receptor antagonism promotes stroke recovery by enhancing axonal plasticity. J Neurosci 2004; 24: 6209-6217.

18. Takahashi M, Vattanajun A, Umeda $T$, Isa K, Isa $T$. Large-scale reorganization of corticofugal fibers after neonatal hemidecortication for functional restoration of forelimb movements. Eur J Neurosci 2009; 30: 1878-1887.

19. Omoto S, Ueno M, Mochio S, Takai T, Yamashita T. Genetic deletion of paired immunoglobulin-like receptor $\mathrm{B}$ does not promote axonal plasticity or functional recovery after traumatic brain injury. J Neurosci 2010; 30: 13045-13052.

20. Baskin YK, Dietrich WD, Green EJ. Two effective behavioral tasks for evaluating sensorimotor dysfunction following traumatic brain injury in mice. $J$ Neurosci Methods 2003; 129: 87-93.

21. Starkey ML, Barritt AW, Yip PK, Davies M, Hamers FP, McMahon SB et al. Assessing behavioural function following a pyramidotomy lesion of the corticospinal tract in adult mice. Exp Neurol 2005; 195: 524-539.

22. Nakamura Y, Fujita Y, Ueno M, Takai T, Yamashita T. Paired immunoglobulin-like receptor $B$ knockout does not enhance axonal regeneration or locomotor recovery after spinal cord injury. J Biol Chem 2011; 286: 1876-1883. 
23. Massa PT, Saha S, Wu C, Jarosinski KW. Expression and function of the protein tyrosine phosphatase SHP-1 in oligodendrocytes. Glia 2000; 29: 376-385.

24. Horvat A, Schwaiger FW, Hager G, Brocker F, Streif R, Knyazev PG et al. A novel role for protein tyrosine phosphatase SHP1 in controlling glial activation in the normal and injured nervous system. J Neurosci 2001; 21: 865-874.

25. Kozlowski M, Mlinaric-Rascan I, Feng GS, Shen R, Pawson T, Siminovitch KA. Expression and catalytic activity of the tyrosine phosphatase PTP1C is severely impaired in motheaten and viable motheaten mice. J Exp Med 1993; 178: 2157-2163.

26. Poole AW, Jones MLA. SHPing tale: perspectives on the regulation of SHP-1 and SHP-2 tyrosine phosphatases by the C-terminal tail. Cell Signal 2005; 17: 1323-1332.

27. Christophi GP, Hudson CA, Gruber R, Christophi CP, Massa PT. Promoter-specific induction of the phosphatase SHP-1 by viral infection and cytokines in CNS glia. J Neurochem 2008; 105: 2511-2523.

28. Zai L, Ferrari C, Subbaiah S, Havton LA, Coppola G, Strittmatter S et al. Inosine alters gene expression and axonal projections in neurons contralateral to a cortical infarct and improves skilled use of the impaired limb. J Neurosci 2009; 29: 8187-8197.

29. Pronichev IV, Lenkov DN. Functional mapping of the motor cortex of the white mouse by a microstimulation method. Neurosci Behav Physiol 1998; 28: 80-85.
30. Thallmair M, Metz GA, Z'Graggen WJ, Raineteau O, Kartje GL, Schwab ME. Neurite growth inhibitors restrict plasticity and functional recovery following corticospinal tract lesions. Nat Neurosci 1998; 1: 124-131.

31. Swanson RA, Morton MT, Tsao-Wu G, Savalos RA, Davidson C, Sharp FR. A semiautomated method for measuring brain infarct volume. J Cereb Blood Flow Metab 1990; 10: 290-293.

32. Mori M, Kose A, Tsujino T, Tanaka C. Immunocytochemical localization of protein kinase C subspecies in the rat spinal cord: light and electron microscopic study. J Comp Neurol 1990; 299: 167-177.

33. Bradbury EJ, Moon LD, Popat RJ, King VR, Bennett GS, Patel PN et al. Chondroitinase $A B C$ promotes functional recovery after spinal cord injury. Nature 2002; 416: 636-640.

(c) (i) (2) (2) Cell Death and Disease is an open-access journal published by Nature Publishing Group. This work is licensed under a Creative Commons Attribution-NonCommercialShareAlike 3.0 Unported License. To view a copy of this license, visit http://creativecommons.org/licenses/by-nc-sa/3.0/

Supplementary Information accompanies this paper on Cell Death and Disease website (http://www.nature.com/cddis) 